\title{
A diabetic milieu promotes OCT4 and NANOG production in human visceral-derived adipose stem cells
}

\author{
P. Dentelli • C. Barale • G. Togliatto • A. Trombetta • \\ C. Olgasi • M. Gili • C. Riganti • M. Toppino • \\ M. F. Brizzi
}

Received: 18 July 2012 /Accepted: 30 August 2012 /Published online: 12 October 2012

(C) Springer-Verlag Berlin Heidelberg 2012

\begin{abstract}
Aims/hypothesis Successful outcomes have been obtained by exploiting adipose-derived stem cells (ASCs) in regenerative medicine. NADPH oxidase (NOX)-generated reactive oxygen species (ROS) are known to control stem cell self-renewal. Several high glucose (HG)-mediated effects depend on NOX-generated ROS. In this study, we investigated whether, and how mechanistically, HG concentrations control ASC fate in patients with diabetes.

Methods ASCs from the visceral adipose tissue of nondiabetic (N-ASCs) and diabetic participants (D-ASCs), identified by surface markers, were counted and evaluated for ROS generation and stem cell properties. Their ability to release soluble factors was assessed by BioPlex analysis. To reproduce an invitro diabetic glucose milieu, N-ASCs were cultured in HG (25 mmol/l) or normal glucose (NG) concentration ( $5 \mathrm{mmol} / \mathrm{l})$, as control. ASC pluripotency was assessed by in vitro study. The $\mathrm{p} 47^{\mathrm{phox}}$ NOX subunit, AKT and octamer-binding transcription factor 4 (OCT4; also known as POU5F1) were knocked down by small-interfering RNA
\end{abstract}

Electronic supplementary material The online version of this article (doi:10.1007/s00125-012-2734-7) contains peer-reviewed but unedited supplementary material, which is available to authorised users.

P. Dentelli and C. Barale contributed equally to this study.

P. Dentelli $\cdot$ C. Barale $\cdot$ G. Togliatto $\cdot$ A. Trombetta $\cdot$ C. Olgasi $\cdot$

M. Gili • M. F. Brizzi $(\bowtie)$

Department of Medical Sciences, University of Torino,

Corso Dogliotti 14,

10126 Torino, Italy

e-mail: mariafelice.brizzi@unito.it

C. Riganti

Department of Oncology, University of Torino,

Torino, Italy

M. Toppino

Department of Surgical Sciences, University of Torino,

Torino, Italy technology. Stem-cell features were evaluated by sphere cluster formation.

Results The ASC number was higher in diabetic patients than in non-diabetic controls. Production of OCT4 and NANOG, stem-cell-specific transcription factors, was upregulated in D-ASCs compared with N-ASCs. Moreover, we found that ROS production and AKT activation drove DASC, but not N-ASC, secretion. When N-ASCs were cultured in vitro in the presence of $\mathrm{HG}$, they also expressed $O C T 4 / N A N O G$ and formed spheres. By knock-down of the p $47^{\text {phox }}$ NOX subunit, AKT and OCT4 we demonstrated that NOX-generated ROS and their downstream signals are crucial for HG-mediated ASC de-differentiation and proinflammatory cytokine production.

Conclusions/interpretation We herein provide a rationale for exploiting D-ASCs in regenerative medicine and/or exploiting HG preconditioning to increase ASCs ex vivo.

Keywords Adipose-derived stem cells $\cdot$ Cytokines $\cdot$ High glucose $\cdot$ Visceral adipose tissue

\begin{tabular}{|c|c|}
\hline \multicolumn{2}{|c|}{ Abbreviations } \\
\hline ASC & Adipose-derived stem cell \\
\hline D-ASCs & $\begin{array}{l}\text { Adipose-derived stem cells from } \\
\text { diabetic participants }\end{array}$ \\
\hline DCF-DA & $\begin{array}{l}\text { 5-(and-6)-Carboxy-2', } 7^{\prime} \text {-dichlorofluorescein } \\
\text { diacetate }\end{array}$ \\
\hline $\mathrm{HG}$ & High glucose \\
\hline HM & High mannitol \\
\hline MSC & Mesenchymal stem cell \\
\hline N-ASCs & $\begin{array}{l}\text { Adipose-derived stem cells from } \\
\text { non-diabetic participants }\end{array}$ \\
\hline NG & Normal glucose \\
\hline NOX & NADPH oxidase \\
\hline OCT4 & Octamer-binding transcription factor 4 \\
\hline PCNA & Proliferating cell nuclear antigen \\
\hline PI3K & Phosphoinositide 3-kinase \\
\hline
\end{tabular}


qRT-PCR Quantitative real-time PCR

ROS Reactive oxygen species

siRNA Small interfering RNA

\section{Introduction}

Human adult mesenchymal stem cells (MSCs), owing to their useful properties, have become of increasing interest to clinicians [1-4]. Indeed, their ability to differentiate into several mesenchymal lineages has spurred clinicians to exploit these cells in tissue engineering and in regenerative medicine to repair damaged organs [1-4]. Different tissues have been discovered as sources of adult stem cells and recently adipose tissue has been considered a novel promising, abundant and accessible resource of adipose-derived stem cells (ASCs) [5-7]. Successful outcomes have been reported from a number of clinical trials exploiting ASCs, mainly in regeneration or repair of failing organs [8], although a number of relevant issues remain to be clarified. In particular, whether the therapeutic effect of ASCs depends merely on their engraftment and differentiation is still debated [9]. Moreover, very few data are as yet available on the molecular mechanisms that regulate their self-renewal and differentiation, both in physiological and pathological settings [10].

The stem cell microenvironment, the 'stem cell niche', in adipose tissue is crucial for preserving ASC self-renewal, denoting 'stemness' potential and controlling differentiation [11]. To date, data have been provided on the negative effects of obesity on the adipogenic niche [10]. However, how mechanistically abnormal metabolic conditions act on niches to control ASC fate is still poorly understood. Likewise, whether and how abnormal metabolic conditions might alter the ability of ASCs to release soluble mediators, a mechanism required for their therapeutic potential [12], is still unknown.

Stem cell pluripotency and differentiation are strictly controlled by a coordinated network of transcription factors [13]. Among them, octamer-binding transcription factor 4 (OCT4) and NANOG, the levels of which are strictly controlled by OCT4 and SRY-box 2 (SOX2) [14], have been long recognised as crucial transcriptional regulators of stem cell self-renewal during embryogenesis [15]. More recently, it has been shown that both OCT4 and NANOG are also produced by adult undifferentiated MSCs and can be recovered from bone marrow, human cord blood and umbilical cord matrix [15-19].

The ability to reciprocally de-differentiate, re-differentiate or trans-differentiate in response to a specific stimulus is defined as cell plasticity [20-22]. OCT4 overexpression has been shown to induce de-differentiation of ASCs into a more immature status by activating the AKT/phosphoinositide 3kinase (PI3K) and extracellular signal-related kinase (ERK1/
2) signalling pathways [23]. As in developmental processes, in adult tissues several factors are engaged to modulate and maintain the balance between self-renewal and differentiation $[21,22]$. Indeed, it has been reported that ASCs possess their own multipotency to reprogramme into more primitive stem cells [23]. However, how this process takes place mechanistically is still controversial.

NADPH oxidase (NOX)-mediated reactive oxygen species (ROS) production [24] has been reported to be involved in the control of stem and progenitor cell fate [25], as well as in neural cell reprogramming [26]. Likewise, ROS generation in response to hypoxia seems to control ASC proliferation [27].

In the diabetic setting, hyperglycaemia, via NOX enzymatic activity, upholds the production of ROS, which in turn acts as a second messenger [28]. Collectively, this might suggest that high glucose (HG) levels, a common feature in diabetes, might also regulate the fate or functional behaviour of stem cells in visceral adipose tissue by activating downstream signals. The present study aimed to investigate whether HG might dictate the balance between self-renewal and differentiation of ASCs in the 'niche' and, if so, how HG acts. In particular, this study aimed to investigate the potential clinical application of ASCs in diabetic patients.

\section{Methods}

Patients and controls A total of 25 type 2 diabetic patients and 15 non-diabetic participants who underwent abdominal surgery (gallbladder removal, in situ colorectal cancer) in our clinic were included. Non-diabetic participants were used as controls. Biometric data from the participants are reported in the electronic supplementary material (ESM) Table 1. Ethics approval was obtained from the Institutional Review Board of S. Giovanni Battista Hospital, Turin, Italy. Informed consent was obtained according to the Helsinki Declaration. We also declare that for the present study, we had no direct contact with the participants.

Isolation of human visceral-derived ASCs Human visceral adipose tissue samples were recovered from 20 type 2 diabetic patients (D-ASCs) and 10 non-diabetic controls (N-ASCs) and processed as detailed in the ESM Methods. To select the ASC subpopulation from the stromal vascular fraction, cells obtained from an additional five patients and five controls were subjected to CD31 and CD45 negative selection by magnetic cell sorting and characterised as detailed in the ESM Methods [29]. Alternatively, visceral NASCs were maintained in normal glucose (NG) DMEM (5 mmol/l D-glucose), HG DMEM (25 mmol/l D-glucose) or high mannitol (HM) DMEM (19 mmol/l D-mannitol, as osmotic control) and processed. Cell viability was evaluated at the end of each experiment using Trypan Blue or MTT assay. 
ASC proliferation Cell proliferation was assayed at different time intervals by direct cell count or FACS analysis (FACSCalibur flow cytometer; BD Biosciences, San Jose, CA, USA) using the proliferating cell nuclear antigen (PCNA) antibody [30]. Alternatively, cell proliferation was performed at day 16 after $48 \mathrm{~h}$ apocynin treatment or $\mathrm{p} 47^{\text {phox }}$ silencing on HG-cultured N-ASCs [31].

Detection of apoptosis Apoptosis was evaluated as indicated by a Cell Death Detection ELISA ${ }^{\text {PLUS }}$ kit (Roche, Mannheim, Germany), following the manufacturer's instructions.

Senescence assay Senescence was evaluated by measuring the acidic $\beta$-galactosidase activity of $\mathrm{N}$-ASCs that were differently cultured, as previously described [32].

ASC characterisation Freshly isolated ASCs, before or after magnetic cell-sorting selection, were characterised by FACS analysis.

Endothelial and adipogenic ASC differentiation Cell multipotency was assessed under appropriate culture conditions.

$R N A$ isolation and quantitative real-time PCR Total RNA was isolated as previously described [31] from freshly or cultured ASCs, differently treated, and from 'spheroids', as indicated. IL6, IL8,TNF $\alpha$ (also known as TNFA) and IL1 $\beta$ (also known as $I L 1 B$ ) mRNA quantification was performed by quantitative real-time PCR (qRT-PCR).

Western blot Cells were lysed and $50 \mu \mathrm{g}$ of protein was subjected to SDS-PAGE, and processed as described [33].

Endogenous $p 4^{p h o x}, O C T 4$ and AKT silencing by small interfering $R N A$ To transiently knock down $\mathrm{p} 47^{\text {phox }}$, OCT4 or AKT duplex small-interfering RNA (siRNA) (Qiagen, Valencia, CA, USA) was used.

Sphere cluster (spheroid) formation ASC de-differentiation status was evaluated by a sphere cluster formation and clonogenic assay. Sphere cluster formation was evaluated by three different operators counting 10 fields at $\times 20$ magnification and reported as number $\pm \mathrm{SD}$ of spheres per field.

ROS detection Cellular ROS level detection on ASCs, cultured and treated as indicated, was performed by using DCF-DA (5-[and-6]-carboxy-2', 7'-dichlorofluorescein diacetate, $0.5 \mu \mathrm{mol} / 1$ final concentration) (Molecular Probes, Invitrogen, Carlsbad, CA, USA) assay.

Mitochondria isolation and measurement of complex I-III activity Cells incubated in the absence or presence of rotenone $(50 \mu \mathrm{mol} / 1$ for $18 \mathrm{~h})$ were lysed and treated, as detailed in the ESM Methods, to recover cytoplasmic mitochondria and measure complex I-III activity.

Cytokine detection Conditioned media from 14 days of NG or HG cultures were collected and processed to detect the levels of cytokines by a BioPlex Array Reader (Bio-Rad Laboratories, Hercules, CA, USA).

Statistical analysis Data are representative of at least three independent experiments, performed in triplicate, unless otherwise indicated. Densitometric analysis was used to calculate the differences in the fold induction of protein levels and normalised, with a statistically significant difference between experimental and control values indicated by $p<0.05$. Western blot panels and relative densitometric histograms reported as 'relative units' in the figures are representative of the results obtained by experiments performed in triplicate $(n=3, n=4$ or $n=5)$. The significance of differences between experimental and control values (indicated by $p<0.05$ ) was calculated using ANOVA with NewmanKeuls and Tukey multicomparison tests. ANOVA multicomparison testing was also used.

All of the methods described above are fully detailed in the ESM Methods. Reagents and antibodies are also reported in the ESM Methods.

\section{Results}

Characterisation of ASCs derived from diabetic patients To investigate whether environmental cues such as hyperglycaemia can control the fate of visceral-derived ASCs, DASCs were compared with N-ASCs. ASC availability was first evaluated. An average (mean \pm SEM) of $9.16 \times 10^{4} \pm$ $9.2 \times 10^{4}$ and $5.95 \times 10^{4} \pm 7.5 \times 10^{4}$ ASCs per gram of visceral adipose tissue were isolated from patients and controls, respectively (Fig. 1a). However, as such a population also contains the stromal vascular fraction, to attain purified ASCs, cells recovered from additional patients and controls (five of each) were subjected to CD31 and CD45 negative selection by magnetic cell sorting [29] and counted. An average (mean \pm SEM) of $4.58 \times 10^{4} \pm 3.2 \times 10^{4}$ and $1.9 \times 10^{4} \pm 2.8 \times$ $10^{4}$ ASCs per gram of visceral adipose tissue were isolated from patients and controls, respectively (ESM Fig. 1a). Cell viability was evaluated by Trypan Blue staining and by MTT assay (data not shown).

Freshly isolated or purified ASCs were subsequently characterised for their expression profile. N-ASCs and D-ASCs, before or after magnetic cell sorting, expressed similar levels of mesenchymal cell markers, such as CD29 and CD44. By contrast, levels of the stemness markers CD105 and CD90 were higher in ASCs from diabetic patients compared with those from non-diabetic controls (Fig. 1b and ESM Fig. 1b). 


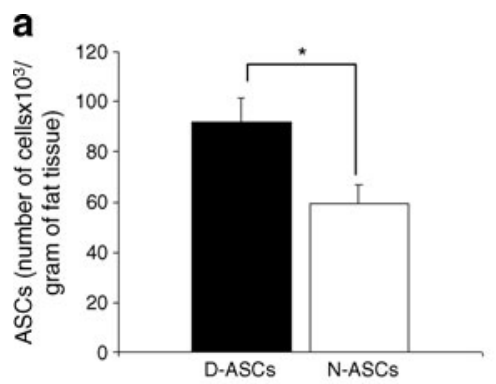

b
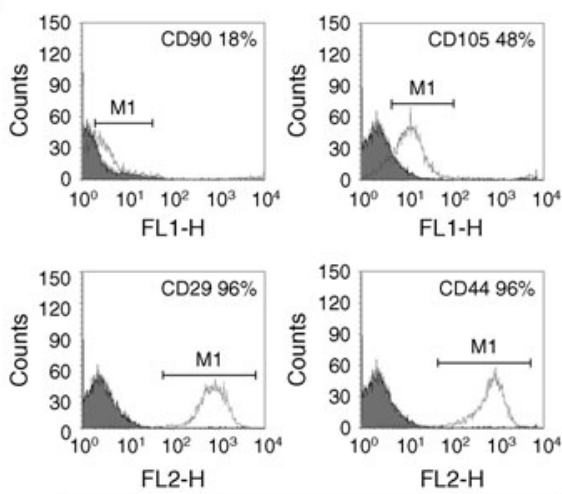

$\mathrm{N}-\mathrm{ASCs}$

Fig. 1 Characterisation of ASCs derived from diabetic patients and non-diabetic controls. (a) The number of visceral ASCs (mean \pm SEM) per gram of fat tissue at isolation. Data refer to type 2 diabetic patients ( $n=20$ samples) or non-diabetic controls $(n=10) .{ }^{*} p<0.05$, D-ASCs vs N-ASCs. (b) Representative FACS analysis of visceral-derived DASCs and N-ASCs soon after isolation. Isotype controls were included (black peaks). The percentage of expression \pm SD of CD44, CD29, CD90 and CD105 markers was obtained by analysing 20 D-ASC and

C
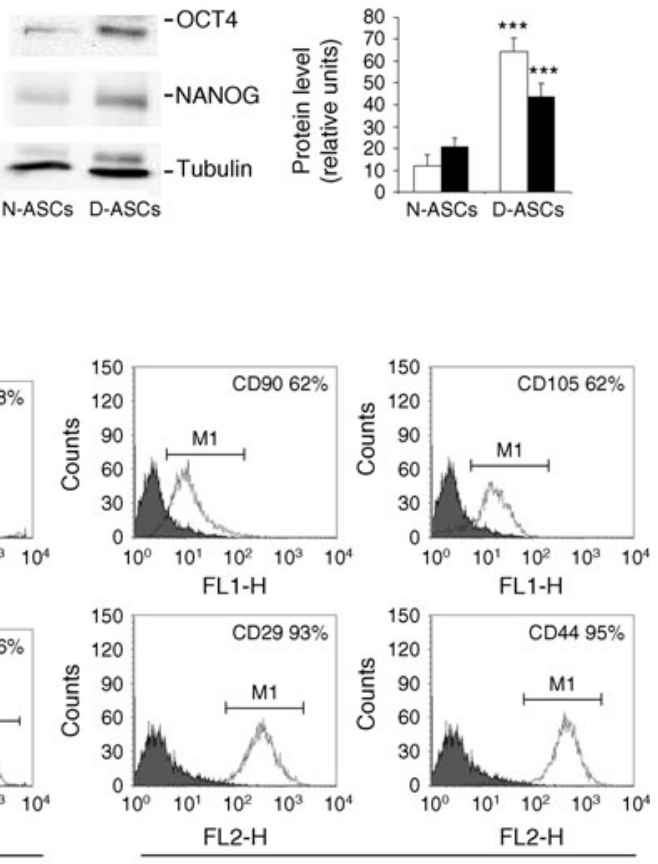

D-ASCs

$10 \mathrm{~N}-A S C$ samples (for N-ASCs: CD44, 96 $\pm 2 \%$; CD29, $96 \pm 2 \%$; CD90, $18 \pm 1 \%$; CD105, 48 $\pm 3 \%$; for D-ASCs: CD44, 95 $\pm 3 \%$; CD29, 93 $\pm 1 \%$; CD90, 62 $\pm 2 \%$; CD105, 62 $\pm 4 \%$ ). (c) Cell extracts from N-ASCs and D-ASCs were analysed by western blot for OCT4 (white bars) and NANOG (black bars) content by densitometry. Protein levels were normalised to tubulin content. The results are representative of three different experiments, each performed in triplicate $(n=3)$. $* * * p<0.001$ vs equivalent group in N-ASCs

higher in freshly recovered D-ASCs than in N-ASCs. To further confirm these results, DCF-DA-fluorescence was also performed on the $\mathrm{CD} 31^{-} / \mathrm{CD} 45^{-} \mathrm{ASC}$ population. Again, ROS generation was higher in D-ASCs than in N-ASCs (ESM Fig. 1d). As hyperglycaemia is a common feature of diabetes, we hypothesised that ASC de-differentiation status could be reproduced in vitro by culturing N-ASCs in $\mathrm{HG}$ conditions. A total of 14 days of culture are required to select a CD31 ${ }^{-} / \mathrm{CD} 45^{-}$ASC population (ESM Fig. 3a) [6]. Thus, the impact of HG in mediating ROS generation was evaluated on freshly recovered ASCs cultured in HG-containing media for 14 days. The results reported in Fig. 2b, obtained by N-ASCs cultured with $\mathrm{HG}$ or NG, demonstrated that ROS production increased in response to $\mathrm{HG}$, but not to NG. Hydrogen peroxide $\left(\mathrm{H}_{2} \mathrm{O}_{2}\right)$-treated cells were used as controls. To investigate the involvement of NOX in ROS generation, $\mathrm{HG}$ or NG cultures were subjected to apocynin treatment. Apocynin treatment was able to prevent HG-generated ROS (Fig. 2c). As apocynin is known to act as a radical scavenger [35] and the assembly of $\mathrm{p} 47^{\text {phox }}$ and $\mathrm{p} 67^{\text {phox }}$ subunits is required for NOX enzymatic activity [36], N-ASCs endogenously depleted of $\mathrm{p} 47^{\text {phox }}$ (ESM Fig. 4a) were evaluated for ROS 
a

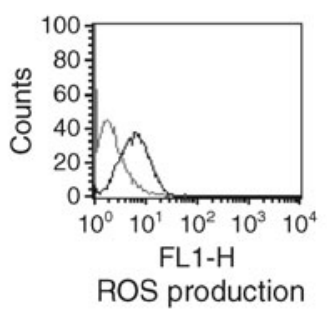

b

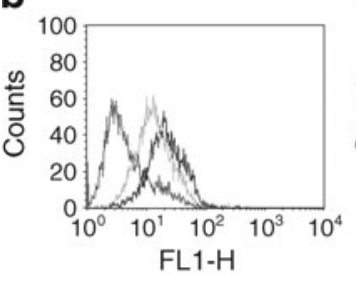

C
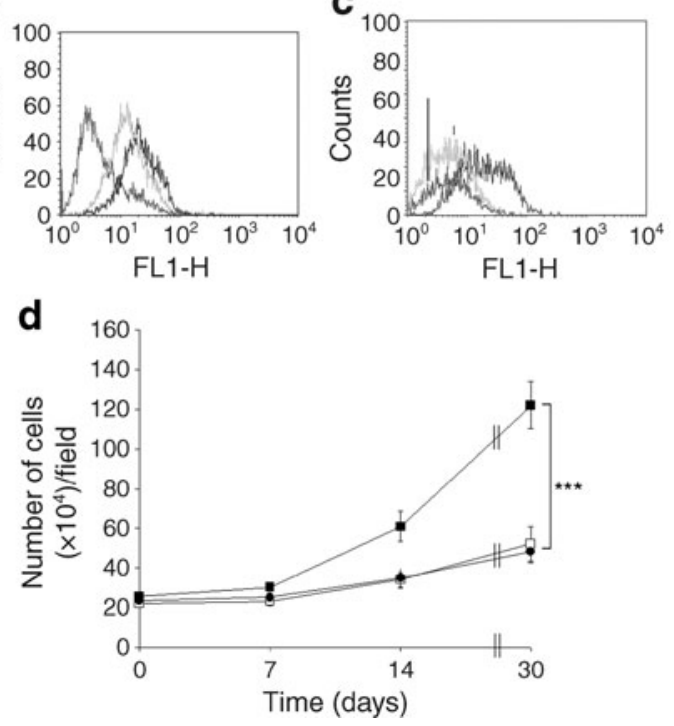

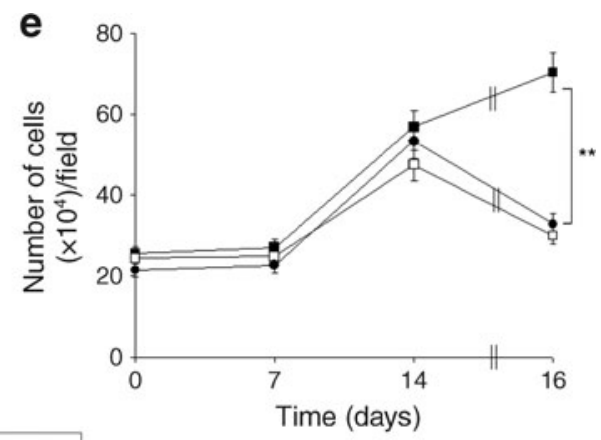

f
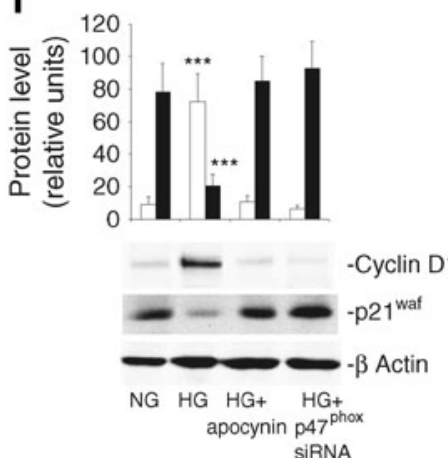

Fig. 2 NOX-mediated ROS production controls ASC proliferation. (a) DCF-DA assay was performed soon after isolation on ASCs freshly recovered from the visceral adipose tissue of type 2 diabetic patients (D-ASCs; black line) or non-diabetic controls (N-ASCs; grey line), to evaluate intracellular ROS levels. The intracellular DCF fluorescence levels, generated by the presence of ROS, varied linearly with increasing concentrations of oxidants and for this reason were used as a marker to quantify intracellular ROS production. The result is representative of three different experiments, each performed in triplicate $(n=3)$. (b) DCF-DA assay was performed on N-ASCs cultured for 14 days in the presence of NG (dark-grey line) or HG (black line), to evaluate ROS generation. $\mathrm{H}_{2} \mathrm{O}_{2}$-treated cells (light-grey line) were used as a positive control for ROS generation. HG-cultured N-ASCs were also treated with apocynin (light-grey line) or depleted of $\mathrm{p} 47^{\text {phox }}$ by siRNA (grey line) and analysed for ROS generation. HG, black line.

generation. As shown in Fig. 2c, knock-down of p4 $7^{\text {phox }}$ prevented ROS production in HG-cultured N-ASCs. To exclude the possibility that mitochondria could contribute to ROS production, the mitochondrial complex I-III inhibitor rotenone [37] was added and ROS generation was evaluated. As shown in ESM Fig. 3b, HG-dependent ROS production was not affected by rotenone treatment. These data were confirmed by measuring complex I-III activity (ESM Fig. 3c). Cell viability was evaluated by MTT assay (data not shown).

NOX-generated ROS are crucial for HG-mediated ASC proliferation To evaluate whether increased ROS levels translate into increased cell proliferation, NG- or HG-cultured (c) Proliferation of visceral N-ASCs, cultured with NG (white squares), HG (black squares) or HM (black circles) $(19 \mathrm{mmol} / 1$, as osmotic control), was evaluated at different time intervals (as indicated) by a direct cell count. ${ }^{* *} p<0.001, \mathrm{~N}-\mathrm{ASC}$ cultured in NG or HM vs HG. (d) HG-cultured N-ASC proliferation was evaluated after apocynin treatment $(48 \mathrm{~h})$ or in cells depleted of $\mathrm{p} 47^{\text {phox }}$ at day 16 by direct cell count (black squares, $\mathrm{HG}$; white squares, $\mathrm{HG}+$ apocynin; black circles, $\mathrm{HG}+\mathrm{p} 47^{\text {phox }}$ siRNA). $* * p<0.01, \mathrm{HG}+$ apocynin and $\mathrm{HG}+\mathrm{p} 47^{\text {phox }}$ siRNA vs HG. Data reported in (c) and (d) are expressed as number \pm SD cells per field ( $\times 10$ magnification). (e) An aliquot of $\mathrm{N}$-ASCs cultured in NG or treated as indicated in panel (d) was analysed by western blot for cyclin D1 (white bars), p2 $1^{\text {waf }}$ (black bars) and $\beta$-actin content; the graph shows densitometric data. The results are representative of three different experiments performed in triplicate $(n=3)$. $* * * p<0.001$ vs equivalent protein in $\mathrm{NG}$

N-ASCs were analysed for their proliferative capability. Data reported in Fig. 2d, obtained by directly counting cells or analysing the abundance of a DNA-polymerase- $\delta$ auxiliary protein such as PCNA (ESM Fig. 5a), revealed the rate of cell proliferation was higher in HG-cultured N-ASCs than in those that were cultured with NG. HM (19 mmol/l), used as osmotic control, had no effect (Fig. 2d and ESM Fig. 5a). MTT assay as well as the cell death detection ELISA assay, used as an index of DNA fragmentation (ESM Fig. 6), revealed that nontoxic ROS levels were generated in response to $\mathrm{HG}$ treatment. To investigate the role of ROS in HG-mediated N-ASC proliferation, HG-cultured N-ASCs were treated with apocynin for $48 \mathrm{~h}$ and evaluated for their ability to undergo cell 
proliferation. As reported in Fig. 2e and ESM Fig. 5b, the addition of apocynin led to a reduction of N-ASC proliferation. The inhibitory effect exerted by apocynin was further confirmed by analysing the content of cyclin D1 (a member of the cyclin protein family that drives the G1/S phase transition) and $\mathrm{p} 21^{\text {waf }}$ (a cyclin-dependent kinase inhibitor acting as a negative regulator of cell cycle progression at G1) (Fig. 2f). Similar results were obtained in N-ASCs silenced for $\mathrm{p} 47^{\text {phox }}$ (Fig. 2e, f and ESM Fig. 5b). The finding that, when depleted of their signalling machinery, ASCs underwent cell senescence could provide an explanation for the reduced numbers of cells detected in these experimental conditions (ESM Fig. 5e). Conversely, both apocynin treatment and $\mathrm{p} 47^{\text {phox }}$ depletion did not affect N-ASC proliferation when cultured in the presence of NG (ESM Fig. 5c and 5d).

$H G$ drives OCT4 and NANOG expression by NOX-mediated ROS generation FACS analysis was performed to characterise HG- and NG-cultured N-ASCs. The data reported in Fig. 3a demonstrate that while N-ASCs cultured with HG for 14 days still contained CD90, those cultured with $\mathrm{NG}$ retained only mesenchymal cell markers $\left(\mathrm{CD} 105^{+}\right.$, $\mathrm{CD} 29^{+}$and $\mathrm{CD}_{4} 4^{+}$). Similarly to D-ASCs, HG-cultured NASCs produced OCT4 and NANOG (Fig. 3b). Undifferentiated adult stem cells, unlike committed cells, grow in suspension and form sphere clusters, in an anchorageindependent fashion [38]. Consistent with OCT4/NANOG content, only HG-cultured N-ASCs were able to form 'spheroids' (Fig. 3c). To investigate the role of ROS in this process, HG-cultured N-ASCs were subjected to apocynin or depleted of $\mathrm{p} 47^{\mathrm{phox}}$ and evaluated. Data reported in Fig. 4a demonstrate that NOX-mediated ROS generation is essential for spheroid formation. Moreover, the finding that both approaches were able to decrease OCT4/NANOG content (Fig. 4b) further sustains the conclusion that ROS generation is crucial to promote ASC de-differentiation. a
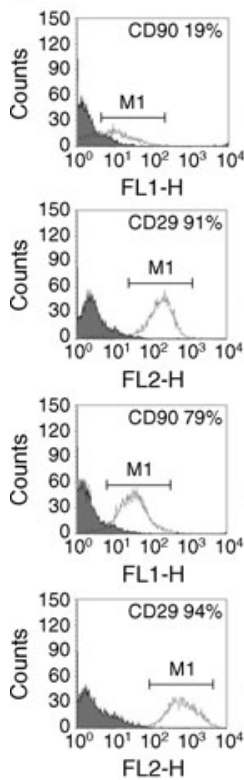

FL2-H b
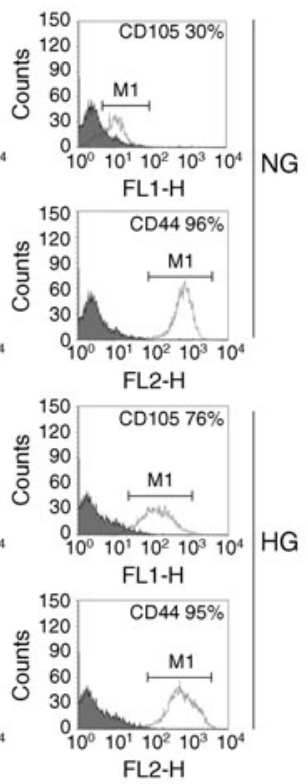

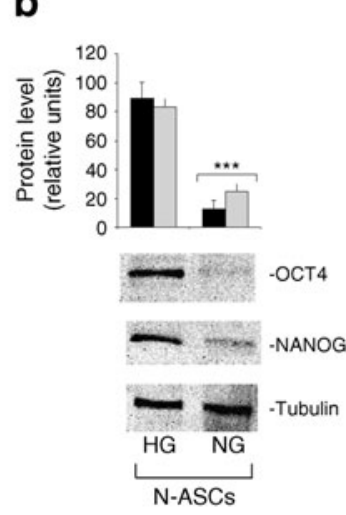

N-ASCs
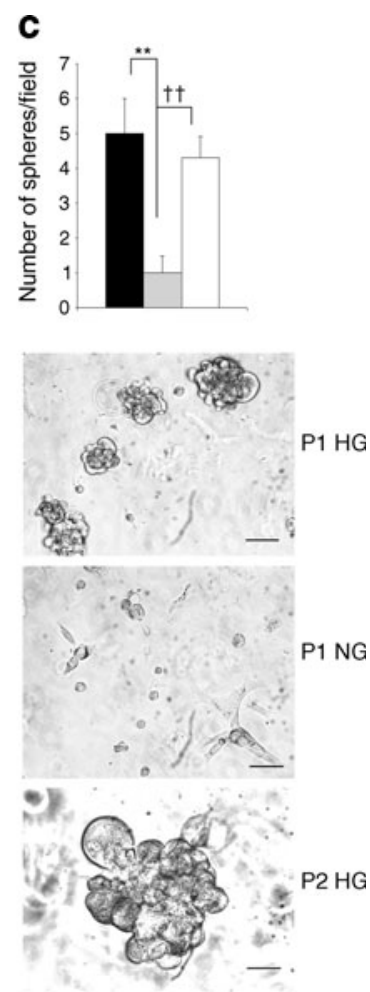

Fig. 3 HG induces OCT4 and NANOG production, as well as sphere cluster formation. (a) Representative FACS analysis of N-ASCs cultured for 14 days in NG or HG medium. Isotype controls were included (black peaks). The percentage of expression $\pm \mathrm{SD}$ of CD44, CD29, CD90 and CD105 markers was obtained by analysing 10 different

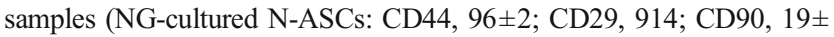
2; CD105, 30 \pm 2; HG-cultured N-ASCs: CD44, 95 \pm 3 ; CD29, 94 \pm 1 ; CD90, 79 \pm 3 ; CD105, 76 \pm 3 ). (b) Cell extracts from N-ASCs culture for 14 days with NG or HG were analysed by western blot for OCT4 (black bars) and NANOG (grey bars) content by densitometry. Protein levels were normalised to tubulin content. ${ }^{* * *} p<0.001$. (c) Sphere cluster formation was evaluated $72 \mathrm{~h}$ after seeding N-ASCs cultured for 14 days in NG or HG in semi-solid medium, as described in the ESM Methods.
Black bar, P1 HG; grey bar, P1 NG; white bar, P2 HG. ** $p<0.01$, P1 NG vs P1 HG. The clonogenic potential of HG-cultured N-ASCs was investigated by plating single cell suspension derived from $\mathrm{P} 1$ spheres for an additional $48 \mathrm{~h}$ (P2 HG). ${ }^{\dagger} p<0.01$, P1 NG vs P2 HG. Sphere cluster formation was evaluated by three different operators (each experiment was performed in triplicate), counting 10 fields at $\times 20$ magnification and reporting as number \pm SD of spheres per field in the histogram. Scale bars indicate $50 \mu \mathrm{m}$ in P1 NG and P1 HG $(\times 20$ magnification $)$ and $20 \mu \mathrm{m}$ in P2 HG ( $\times 40$ magnification). The results are representative of four individual experiments performed in triplicate $(n=4) \mathrm{P} 1$ corresponds to sphere cluster formation; P2 corresponds to sphere cluster formation of P1-derived cells 
a

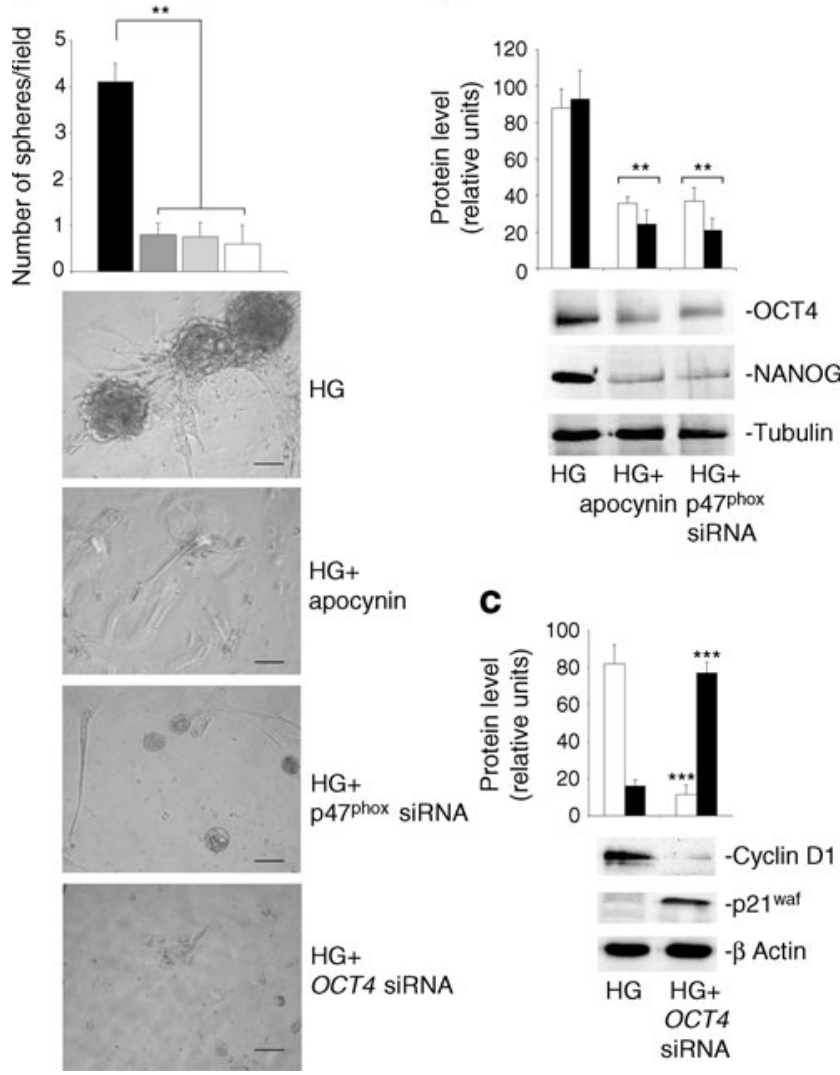

Fig. 4 NOX-mediated ROS generation in response to HG is crucial for both sphere cluster formation and OCT4/NANOG production. (a) NASCs, subjected to the indicated treatments, were plated in semi-solid medium, as described in the ESM Methods, to assess sphere cluster formation. The histogram reports the number $\pm \mathrm{SD}$ of spheres per field, counting 10 fields at $\times 20$ magnification. Black bar, HG; dark-grey bar, $\mathrm{HG}+$ apocynin; light-grey bar, $\mathrm{HG}+\mathrm{p} 47^{\text {phox }}$ siRNA; white bar, $\mathrm{HG}+$ OCT4 siRNA. Scale bars indicate $50 \mu \mathrm{m} .{ }^{* *} p<0.01$. The results are representative of four different experiments performed in triplicate $(n=$ 4). (b) HG-cultured N-ASCs in the presence or absence of apocynin or depleted of $\mathrm{p} 47^{\text {phox }}$ were lysed and subjected to SDS-PAGE. White bars, OCT4; black bars, NANOG. $* * p<0.01$. The filters were immunoblotted with anti-OCT4, anti-NANOG and anti-tubulin antibodies. (c) Cell extracts from HG-cultured N-ASCs, depleted or not of OCT4, were analysed by western blot for cyclin D1 (white bars) and $\mathrm{p} 21^{\text {waf }}$ (black bars) content. Protein levels were normalised to $\beta$-actin content. $* * * p<0.001, \mathrm{HG}+$ OCT4 siRNA-treated cells vs HG for cyclin D1 and $\mathrm{p} 21^{\text {waf }}$ content. The results are representative of three different experiments performed in triplicate $(n=3)$

OCT4 is crucial for HG-mediated ASC de-differentiation ASCs overexpressing OCT4 have been shown to undergo cell reprogramming [23]. Thus, the involvement of OCT4 in controlling HG-mediated ASC de-differentiation was further investigated. As reported in Fig. 4a, knock-down of OCT4 (ESM Fig. 4b) prevented HG-induced sphere cluster formation. Consistently, decreased expression of cyclin D1 and increased expression of $\mathrm{p} 21^{\mathrm{waf}}$ was detected (Fig. 4c) in OCT4-silenced cells.
AKT activation is crucial for OCT4 and NANOG expression and for spheroid formation in response to $H G$ AKT is crucial for OCT4-mediated de-differentiation [23], thus, the role of AKT in HG-mediated ASC reprogramming was investigated. As shown in Fig. 5a, AKT production could be detected in HG-cultured N-ASCs, but not in NG-cultured cells. To gain further insight into the role of AKT in regulating this process, functional studies were performed in AKT-silenced N-ASCs (ESM Fig. 4c). Data in Fig. 5b, c show that, as in apocynin-treated and $\mathrm{p} 47^{\text {phox }}$-silenced $\mathrm{N}$ ASCs, OCT4/NANOG content was reduced and spheroid formation prevented in N-ASCs endogenously depleted of AKT. Again, the content of cyclin D1 and $\mathrm{p} 21^{\text {waf }}$ validated these data (Fig. 5d). To further confirm the role of ROS in controlling AKT activation, N-ASCs were cultured in the presence of apocynin or depleted of $\mathrm{p} 47^{\text {phox }}$. As reported in Fig. 5a in both culture conditions we failed to detect AKT activation. The finding that ROS generation was still detectable in AKT-depleted cells (Fig. 5e) indicates that ROS generation occurs upstream to AKT.

ROS production and AKT activation are required for IL-6 and IL-8 production by spheroids The role of ROS in mediating ASC paracrine action has been reported [39]. Therefore, the contribution of ROS in mediating HG-induced soluble factor secretion was investigated. BioPlex analysis (on supernatant fractions) and qRT-PCR (on spheroids) were performed. As reported in Fig. 6, the amount of IL-6 and IL8 produced by N-ASCs cultured in the presence of $\mathrm{HG}$ (Fig. 6a) and spheroids (Fig. 6c,e) was higher than in NGcultured cells. Next, N-ASC cytokine production was analysed in $\mathrm{p} 47^{\text {phox }}$ and AKT-silenced cells. Data shown in Fig. 6 clearly demonstrate that NOX-generated ROS and AKT activation are required for IL-6 and IL-8 production in response to $\mathrm{HG}$ only, indicating that different mechanisms account for IL-6 and IL-8 production in NG-cultured NASCs (ESM Fig. 7a). Moreover, the finding that preventing spheroid formation by silencing $\mathrm{p} 47^{\text {phox }}$ and $A K T$ led to reduced cytokine production sustains the possibility that $\mathrm{N}$-ASC paracrine function is enhanced by their dedifferentiation status. Consistent with this possibility, we found that the release of IL-6 and IL- 8 was significantly reduced when HG-containing media were removed and replaced with NG (Fig. 6g). Finally, we failed to detect TNF $\alpha$ and IL-1 $\beta$ in our samples (Fig. 6b,d,f).

ROS generation and AKT activation are required for $D-A S C$ cytokine production An increased level of proinflammatory cytokines is a common feature in diabetes [40]. Thus, we investigated whether D-ASC de-differentiation status might contribute to this proinflammatory profile. Soon after isolation, both freshly recovered and $\mathrm{CD} 31^{-} / \mathrm{CD} 45^{-}$ ASCs were evaluated for their ability to produce IL-6 and 

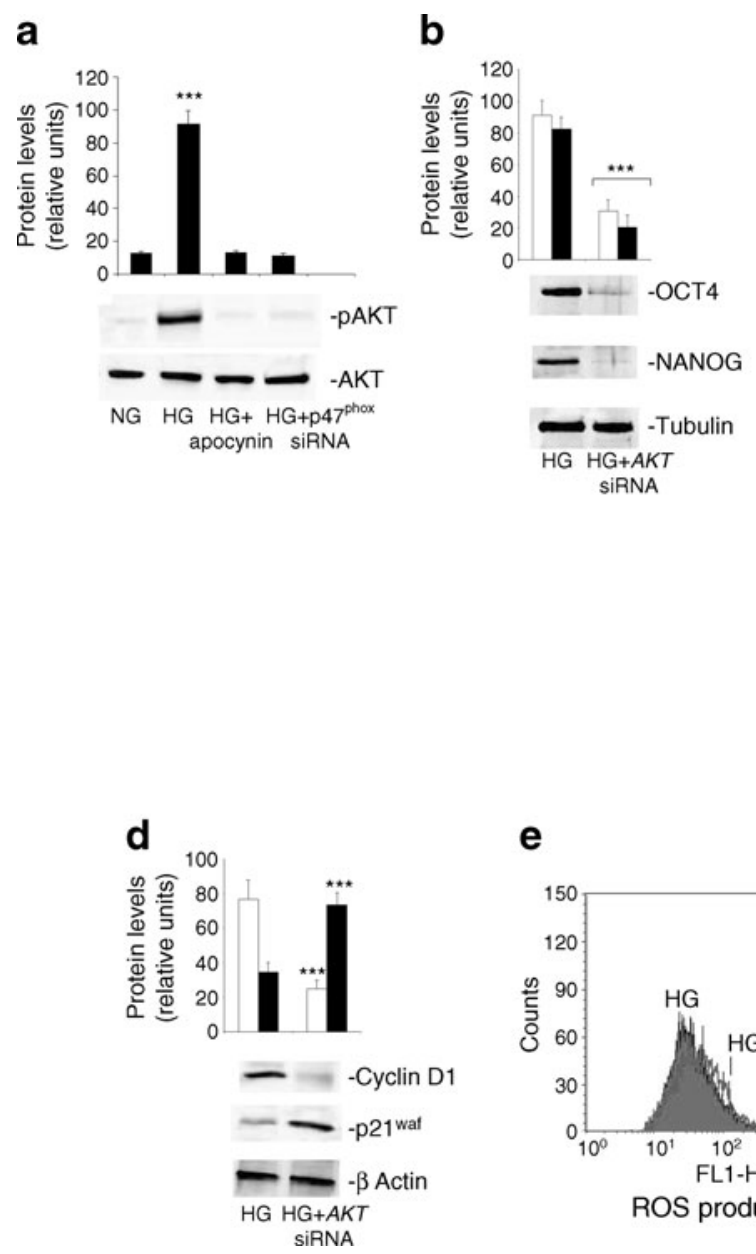

e

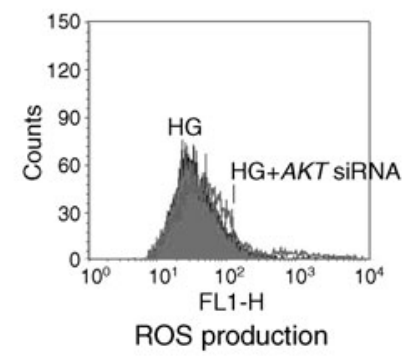

Fig. 5 ROS-mediated AKT activation is involved in sphere cluster formation and OCT4/NANOG production in response to HG. (a) NASCs cultured in different experimental conditions, as indicated, were lysed and analysed by western blot for pAKT (black bars) and normalised to total AKT content. (b) Cell lysates from HG-cultured N-ASCs endogenously depleted of AKT were subjected to SDS-PAGE. The filters were immunoblotted with anti-OCT4 and anti-NANOG antibodies. Protein levels were normalised to tubulin content. White bars, OCT4; black bars, NANOG. (c) Sphere cluster formation assay was performed by seeding HG-cultured N-ASCs untreated or silenced for

IL-8 by qRT-PCR. As reported in Fig. 7 and ESM Fig. 7b, IL-6 and IL-8 production was higher in D-ASCs than in $\mathrm{N}-\mathrm{ASCs}$ in both conditions. To gain further insight into the mechanisms regulating proinflammatory cytokine production and to avoid long-term culture conditions, apocynin and the specific AKT inhibitor TCL1 $1_{10-24}$ were used instead of siRNA technology. Indeed, by applying these approaches, we demonstrated that ROS generation and AKT activation are essential for IL-6 and IL-8 production (Fig. 7 and ESM Fig. 7b). Again, we failed to detect either $\mathrm{TNF} \alpha$ or IL-1 $\beta$.

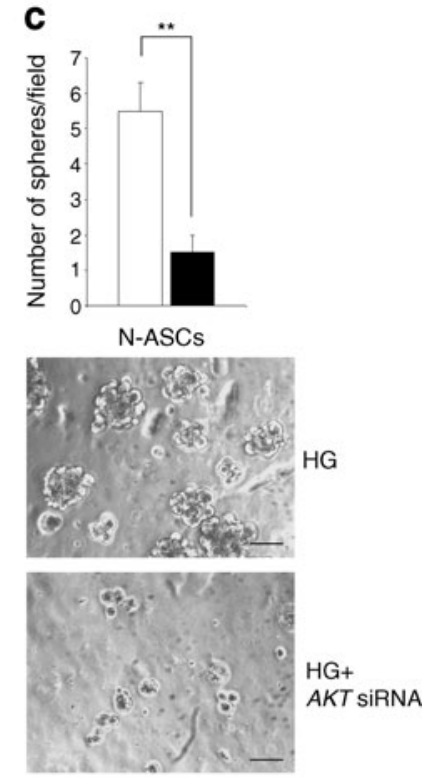

ROS production

AKT in semi-solid medium. Histogram reports the number \pm SD of spheres per field, counting 10 fields at $\times 20$ magnification. Scale bars indicate $50 \mu \mathrm{m}$. ${ }^{*} p<0.01, \mathrm{HG}+A K T$ siRNA (black bar) vs HG (white bar). (d) N-ASCs cultured and treated as indicated above were analysed by western blot for cyclin D1 (white bars), p2 $1^{\text {waf }}$ (black bars) and $\beta$-actin content. (e) DCF-DA assay was performed on HG-cultured N-ASCs, silenced or not for AKT, to evaluate cellular ROS levels. The results are representative of three different experiments, each performed in triplicate $(n=3) . * * * p<0.001$

\section{Discussion}

White adipose tissue, owing to the easy access by minimally invasive methods, is currently considered an alternative and valuable source of ASCs for tissue regeneration $[5,7,41]$. However, no data have been provided on how mechanistically the balance between self-renewal and differentiation in the 'niche' is regulated in pathological conditions. Likewise, studies evaluating the potential impact of ASCs in clinical settings where tissue regeneration is required are still unavailable. 
a

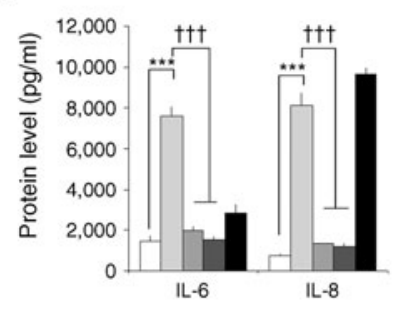

C

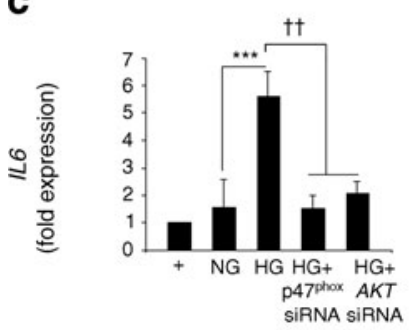

e

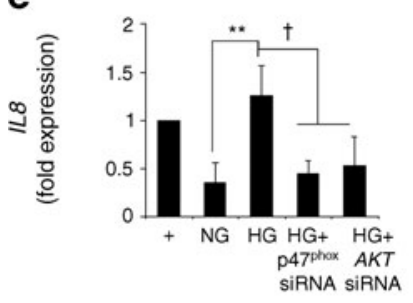

b

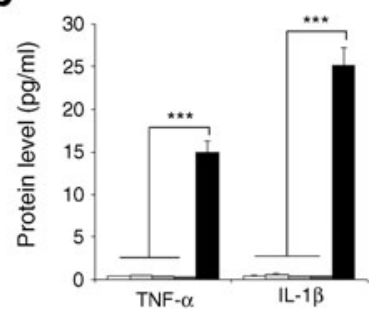

d

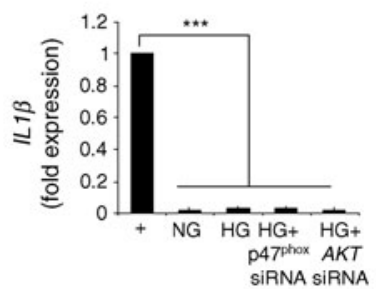

f

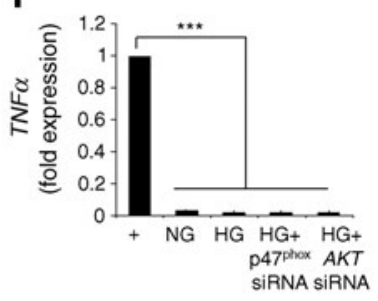

g

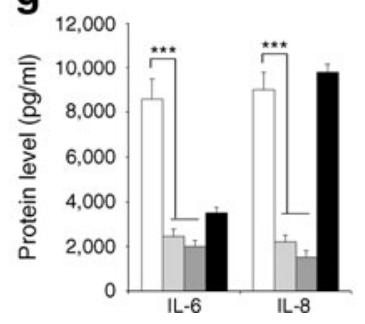

The 'stem cell niche' is a specialised microenvironment containing stem cells [42]. As in developmental processes, in adult tissues, the balance between self-renewal and differentiation of stem cells is strictly controlled by environmental cues [13-18, 21, 22]. In this study, we have demonstrated that hyperglycaemic conditions induce and maintain ASC pluripotency. Pluripotency factors, including stemness genes, provide fundamental mechanisms underlying the properties of stem cells [22]. OCT4, SOX2 and NANOG are known regulators of genes encoding molecules involved in the control of stem cell behaviours, including proliferation and differentiation $[18,21,22]$. We provide evidence that in D-ASCs, OCT4 and NANOG expression is upregulated compared with N-ASCs. Consistently, we found that HG-induced OCT4 and NANOG expression is associated with an increased rate of cell proliferation and with their de-differentiation status. siRNA-mediated gene

4Fig. 6 ROS generation and AKT activation are required for IL-6 and IL-8 production. $(\mathbf{a}, \mathbf{b})$ BioPlex analysis was performed, according to the manufacturer's instructions, to detect IL-6, IL-8 (a) and TNF $\alpha$ and IL-1 $\beta$ (b) levels in sixfold-concentrated supernatant fractions, recovered from the indicated cultures. White bars, NG; light-grey bars, HG; grey bars, $\mathrm{HG}+\mathrm{p} 47^{\text {phox }}$ siRNA; dark-grey bars, $\mathrm{HG}+A K T$ siRNA; black bars, positive control. IL- 6 or IL- 8 : ${ }^{* * *} p<0.001$, HG-cultured N-ASCs vs NG-cultured N-ASCs, ${ }^{\dagger \dagger} p<0.001, \mathrm{HG}+\mathrm{p} 47^{\text {phox }}$ siRNA and $\mathrm{HG}+A K T$ siRNA vs HG. TNF $\alpha$ and IL- $1 \beta$ : $* * * p<0.001$, experimental vs control value. (c-f) $I L 6(\mathbf{c}), I L 1 \beta$ (d), IL8 (e) and $T N F \alpha(\mathbf{f})$ mRNA quantification was performed by qRT-PCR on N-ASCs pretreated as indicated and cultured in semi-solid medium (see sphere cluster formation assay in the ESM Methods). Fold expression of the indicated cytokines, normalised to $G A P D H$, is representative of ten samples. ${ }^{* * *} p<0.001$, HG-cultured N-ASCs vs NG-cultured N-ASCs; ${ }^{\dagger} p<0.01, \mathrm{HG}+\mathrm{p} 47^{\text {phox }}$ siRNA and $\mathrm{HG}+A K T$ siRNA vs HG for IL6; $* * p<0.01$, HG-cultured N-ASCs vs NG-cultured N-ASCs; ${ }^{\dagger} p<0.05$, $\mathrm{HG}+\mathrm{p} 47^{\text {hox }}$ siRNA and $\mathrm{HG}+A K T$ siRNA vs HG for IL8; ***p< 0.001, experimental vs control value for $T N F \alpha$ and $I L I \beta$. (g) BioPlex analysis was also performed to detect IL-6 and IL-8 levels on sixfold concentrated supernatant fractions, recovered from 14 days' NG (darkgrey bars) and HG (white grey bars) cultures. Alternatively, HG medium was replaced for $48 \mathrm{~h}$ with NG medium (light-grey bars) and then collected to detect IL- 6 and IL- $8(* * * p<0.001$, NG and HG replaced with NG vs HG-cultured N-ASCs). Black bars, positive control. In (ad), supernatant fractions or mRNA obtained from activated macrophages were used as positive controls $(+)$. The results in a and $\mathbf{d}$ are representative of three different experiments, each performed in triplicate $(n=3)$

knock-down, used to extend our knowledge on D-ASC stemness behaviour, further supports the role of OCT4 in promoting ASC de-differentiation. This suggests that the increased number of ASCs recovered from diabetic patients may be the result of $\mathrm{HG}$ concentrations in the adipose 'stem cell niche'.

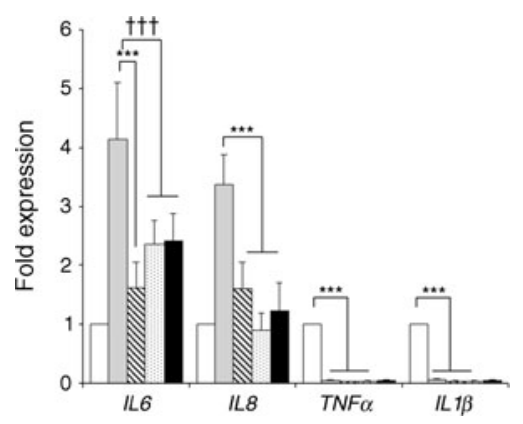

Fig. 7 IL6 and IL8 expression by $\mathrm{CD} 31^{-} / \mathrm{CD} 45^{-}$selected ASCs. IL6, $I L 8, T N F \alpha$ and $I L 1 \beta$ mRNA quantification was performed by qRTPCR on $\mathrm{CD} 31^{-} / \mathrm{CD} 45^{-} \mathrm{N}-\mathrm{ASCs}$ (grey bars) and D-ASCs (striped bars). CD $31^{-} / \mathrm{CD} 45^{-} \mathrm{D}$-ASCs were also treated for $6 \mathrm{~h}$ with apocynin (dotted bars) or with the AKT inhibitor TCL1 $1_{10-24}$ (black bars). Fold expression of the indicated cytokines, normalised to $G A P D H$, is representative of five samples tested. $* * * p<0.001$, D-ASCs vs N-ASCs; ${ }^{\dagger \dagger} p<0.001, \mathrm{D}-\mathrm{ASC}+$ apocynin and D-ASCs$+\mathrm{TCL}_{10-24}$ Vs D-ASCs for IL6; $* * * p<0.01, \mathrm{~N}$-ASCs, D-ASCs+apocynin and D-ASCs+ TCL1 $_{10-24}$ vs D-ASCs for $I L 8 ; * * * p<0.001$, experimental vs control value for $T N F \alpha$ and $I L 1 \beta$. mRNA from activated macrophages was used as positive control (white bars, + ). The results are representative of five different experiments, each performed in triplicate $(n=5)$ 
Although cellular accumulation of ROS has been considered a crucial driver of cell death in different pathological settings [43], evidence has been provided that the reductionoxidation balance plays a far more complex role in stem cell biology $[26,27,44]$. Our data demonstrated that HG challenge induces 'non-toxic' levels of ROS in ASCs. ROS generation mainly relies on NOX enzymatic activity [28], which in turn depends on its regulatory subunits, $\mathrm{p} 47^{\text {phox }}$ and $\mathrm{p} 67^{\text {phox }}[28]$. The finding that $\mathrm{p} 47^{\text {phox }}$ knock-down prevented both ROS generation and ASC proliferation indicates that NOX-mediated ROS generation in response to $\mathrm{HG}$ acts as second messenger to drive ASC proliferation. Moreover, the observation that interfering with the mitochondrial electron transport system did not affect ROS production suggests that ROS generation in our experimental conditions mainly depends on NOX activity. We could not directly demonstrate the involvement of NOX in mediating ROS production in freshly isolated ASCs (to obtain $\mathrm{p} 47^{\text {phox }}$ endogenous depletion would require long-term culture conditions). However, the percentage of $\mathrm{HbA}_{1 \mathrm{c}}$ detected in our patients leads us to speculate that, as in vitro, in D-ASC NOX might be crucial in driving ROS generation, eventually leading to the increased de-differentiation status.

To increase stem cell proliferation and self-renewal potential, physiological levels of ROS are required [25, 26, 45]. Consistently, data provided by our study (demonstrating that by silencing $\mathrm{p} 47^{\text {phox }}$, ASCs failed to form spheroids and did not express OCT4) indicate that NOX-generated ROS are also crucial for HG-driven ASC de-differentiation. Moreover, the possibility that a decrease in ROS cellular levels has a negative impact on ASC self-renewal is supported by the finding that NG-cultured N-ASCs were unable to form spheroids.

Beside phagocytes, other cell types use NOX-generated ROS as a second messenger [46]. The activation of the MAPK and PI3K/AKT signalling pathways has been reported to be the result of NOX-generated ROS [27]. Le Belle et al have demonstrated that in neural stem cell populations, self-renewal is associated with the ROS-mediated PI3K/AKT signalling pathway [26]. In addition, it has been shown that ASC reprogramming induced by OCT4 overexpression engages the PI3K/AKT and JAK/STAT3 pathways [23]. By applying siRNA technology to knock-down NOX and AKT, we have demonstrated that AKT activation is also essential for HG-mediated OCT4/NANOG production and for spheroid formation. Moreover, data provided by knock-down AKT demonstrate that AKT is downstream to ROS and upstream to OCT4. Again, further support for the biological relevance of the AKT signalling pathway in driving HG-mediated ASC de-differentiation is provided by data obtained from NG-cultured N-ASCs.

ASCs, besides possessing the ability to regenerate damaged tissue, also secrete soluble mediators $[12,39,47]$. A great deal of interest in adipose tissue has been generated by the finding that ASCs, by means of their paracrine function, influence the inflammatory response [39, 47]. We demonstrate that microenvironmental cues, by inducing ASC de-differentiation, influence ASC functional behaviour, resulting in increased proinflammatory cytokine production. This suggests that ASCs, exposed to hyperglycaemic conditions via NOX-generated ROS and AKT activation, might actively participate in dysfunctional proinflammatory cytokine production. Epidemiological studies have described a clear link between the $\mathrm{C}$-reactive protein-mediated inflammatory response, its inducer IL- 6 and an increased cardiovascular risk profile [48]. Interventions aimed at ameliorating the metabolic profile lead to a reduction in proinflammatory protein plasma levels, including that of IL-6 [49]. Consistently, our results show that restoring glucose concentrations leads to decreased IL- 6 and IL- 8 production. Therefore, as particular attention has been dedicated to the detrimental consequences of hyperglycaemia, our data suggest that targeting hyperglycaemia could also represent a rational approach to interfering with the ASC dysfunctional cytokine profile.

Adipose tissue transplantation has been proposed as a potential tool to provide favourable metabolic effects in diabetes [3]. Data provided herein open new perspectives for the clinical application of visceral ASCs in diabetic patients. The potential clinical impact is supported by the following observations. First, the number of ASCs recovered from the visceral adipose tissue of diabetic patients is increased compared with that of non-diabetic controls. Second, ASCs obtained from these patients have high content of OCT4/NANOG, and thus possess a wider therapeutic potential. Third, visceral adipose tissue could be an ideal source of autologous ASCs obtainable with minimally invasive procedures. Finally, consistent with data provided by omentectomy in humans [50], the removal of visceral adipose tissue, besides providing favourable metabolic effects, might also offer an additional advantage such as the possibility of exploiting ASCs to repair failing organs. However, we cannot exclude that as in hematopoietic systems, where a high ROS level is associated with a high proliferation rate and premature exhaustion of the self-renewal potential [25], HG concentrations in the visceral adipose tissue of diabetic patients could lead to a rapid exhaustion of ASC stemness capability, eventually translating in an accelerated impairment of the regenerative potential. Further studies are required to resolve this issue.

Data have indicated that multipotent stem cells can be recovered from the adipose tissue of adults [8]. However, how metabolic conditions can modulate the balance between self-renewal and differentiation in the 'niche' is still unclear. In this study, we provide evidence that environmental cues impact ASC biological properties and significantly affect their stemness and secretion profile. Moreover, these results 
provide the rationale to develop therapeutic strategies exploiting adipose tissue as an easily accessible source of MSCs to repair failing organs in diabetic patients.

Acknowledgements We thank Prof. D. Ghigo (Department of Oncology, University of Torino, Torino, Italy) for his helpful advice.

Funding This work was supported by grants from the Italian Association for Cancer Research (AIRC), Ricerca Finalizzata Regione Piemonte and MIUR (Ministero dell'Università e Ricerca Scientifica, cofinanziamento MURST) to MFB.

Duality of interest The authors declare that there is no duality of interest associated with this manuscript.

Contribution statement $\mathrm{PD}, \mathrm{CB}, \mathrm{GT}$ and $\mathrm{AT}$ contributed to the study design, analysis and interpretation of data and to the critical revision of the manuscript. $\mathrm{CO}, \mathrm{MG}$ and $\mathrm{CR}$ contributed to the analysis and interpretation of data, as well as to the revision of the manuscript. MT contributed to the acquisition of data and critical revision of the manuscript. MFB contributed to the study conception and design, and in writing the manuscript. All authors approved the version to be published.

\section{References}

1. Schaffler A, Buchler C (2007) Concise review: adipose tissuederived stromal cells-basic and clinical implications for novel cell-based therapies. Stem Cells 25:818-827

2. Le Blanc K, Ringdén O (2007) Immunomodulation by mesenchymal stem cells and clinical experience. J Intern Med 262:509-525

3. Tran TT, Kahn CR (2010) Transplantation of adipose tissue and stem cells: role in metabolism and disease. Nat Rev Endocrinol 6:195-213

4. Rubina K, Kalinina N, Efimenko A et al (2009) Adipose stromal cells stimulate angiogenesis via promoting progenitor cell differentiation, secretion of angiogenic factors, and enhancing vessel maturation. Tissue Eng Part A 15:2039-2050

5. Zuk PA, Zhu M, Ashjian P et al (2002) Human adipose tissue is a source of multipotent stem cells. Mol Biol Cell 13:4279-4295

6. Baglioni S, Francalanci M, Squecco R et al (2009) Characterization of human adult stem-cell populations isolated from visceral and subcutaneous adipose tissue. FASEB J 23:3494-3505

7. Gimble JM, Katz JA, Bunnell BA (2007) Adipose derived stem cells for regenerative medicine. Circ Res 100:1249-1260

8. Locke M, Feisst V, Dunbar PR (2011) Concise review: human adipose-derived stem cells: separating promise from clinical need. Stem Cells 29:404-411

9. Yarak S, Okamoto OK (2010) Human adipose-derived stem cells: current challenges and clinical perspectives. An Bras Dermatol 85:647-656

10. Roldan M, Macias-Gonzalez M, Garcia R, Tinahones FJ, Martin M (2011) Obesity short-circuits stemness gene network in human adipose multipotent stem cells. FASEB J 25:4111-4126

11. Schraufstatter IU, Discipio RG, Khaldoyanidi S (2011) Mesenchymal stem cells and their microenvironment. Front Biosci 17:22712288

12. Horwitz EM, Dominici M (2008) How do mesenchymal stromal cells exert their therapeutic benefit? Cytotherapy 10:771-774
13. Ng HH, Surani MA (2011) The transcriptional and signalling networks of pluripotency. Nat Cell Biol 13:490-496

14. Kuroda T, Tada M, Kubota $\mathrm{H}$ et al (2005) Octamer and Sox elements are required for transcriptional cis regulation of NANOG gene expression. Mol Cell Biol 25:2475-2485

15. Loh YH, Wu Q, Chew JL et al (2006) The OCT4 and NANOG transcription network regulates pluripotency in mouse embryonic stem cells. Nat Genet 38:431-440

16. Silva J, Chambers I, Pollard S, Smith A (2006) NANOG promotes transfer of pluripotency after cell fusion. Nature 441:997-1001

17. Lengner CJ, Welstead GG, Jaenisch R (2008) The pluripotency regulator OCT4: a role in somatic stem cells? Cell Cycle 7:725728

18. Park IH, Zhao R, West JA et al (2008) Reprogramming of human somatic cells to pluripotency with defined factors. Nature 451:141146

19. Santourlidis S, Wernet $P$, Ghanjati $F$ et al (2011) Unrestricted somatic stem cells (USSC) from human umbilical cord blood display uncommitted epigenetic signatures of the major stem cell pluripotency genes. Stem Cell Res 6:60-69

20. Freberg CT, Dahl JA, Timoskainen S, Collas P (2007) Epigenetic reprogramming of OCT4 and NANOG regulatory regions by embryonal carcinoma cell extract. Mol Biol Cell 18:1543-1553

21. Goodell MA (2003) Stem-cell "plasticity": befuddled by the muddle. Curr Opin Hematol 10:208-213

22. Sanges D, Cosma MP (2010) Reprogramming cell fate to pluripotency: the decision-making signalling pathways. Int J Dev Biol 54:1575-1587

23. Kim JH, Jee MK, Lee SY et al (2009) Regulation of adipose tissue stromal cells behaviors by endogenic OCT4 expression control. PLoS One 4:e7166

24. Lambeth JD, Krause KH, Clark RA (2008) NOX enzymes as novel targets for drug development. Semin Immunopathol 30:339363

25. Jang YY, Sharkis SJ (2007) A low level of reactive oxygen species selects for primitive hematopoietic stem cells that may reside in the low-oxygenic niche. Blood 110:3056-3063

26. Le Belle JE, Orozco NM, Paucar AA et al (2011) Proliferative neural stem cells have high endogenous ROS levels that regulate self-renewal and neurogenesis in a PI3K/AKT-dependant manner. Cell Stem Cell 8:59-71

27. Kim JH, Park SH, Park SG, Choi JS, Xia Y, Sung JH (2011) The pivotal role of reactive oxygen species generation in the hypoxiainduced stimulation of adipose-derived stem cells. Stem Cells Dev 20:1753-1761

28. Kakehi T, Yabe-Nishimura (2008) NOX enzymes and diabetic complications. Semin Immunopathol C 30:301-314

29. Zhang P, Moudgill N, Hager E et al (2011) Endothelial differentiation of adipose-derived stem cells from elderly patients with cardiovascular disease. Stem Cells Dev 20:977-988

30. Dentelli P, Rosso A, Olgasi C, Camussi G, Brizzi MF (2011) IL-3 is a novel target to interfere with tumor vasculature. Oncogene 30:4930-4940

31. Togliatto G, Trombetta A, Dentelli P, Rosso A, Brizzi MF (2011) MIR221/MIR222-driven post-transcriptional regulation of P27KIP1 and P57KIP2 is crucial for high-glucose- and AGE-mediated vascular cell damage. Diabetologia 54:1930-1940

32. Togliatto G, Trombetta A, Dentelli P et al (2010) Unacylated ghrelin rescues endothelial progenitor cell function in individuals with type 2 diabetes. Diabetes 59:1016-1025

33. Dentelli P, Trombetta A, Togliatto G et al (2009) Formation of STAT5/PPARgamma transcriptional complex modulates angiogenic cell bioavailability in diabetes. Arterioscler Thromb Vasc Biol 29:114-120

34. Liu TM, Martina M, Hutmacher DW, Hui JH, Lee EH, Lim B (2007) Identification of common pathways mediating 
differentiation of bone marrow- and adipose tissue-derived human mesenchymal stem cells into three mesenchymal lineages. Stem Cells 25:750-760

35. Heumüller S, Sven W, Barbosa-Sicard E et al (2008) Apocynin is not an inhibitor of vascular NADPH oxidases but an antioxidant. Hypertension 51:211-217

36. Babior BM (2004) NADPH oxidase. Curr Opin Immunol 16:42-47

37. Brand MD, Affourtit C, Esteves TC et al (2004) Mitochondrial superoxide: production, biological effects, and activation of uncoupling proteins. Free Radic Biol Med 37:755-767

38. Reynolds BA, Weiss S (1996) Clonal and population analyses demonstrate that an EGF-responsive mammalian embryonic CNS precursor is a stem cell. Dev Biol 175:1-13

39. Carrière A, Ebrahimian TG, Dehez S et al (2009) Preconditioning by mitochondrial reactive oxygen species improves the proangiogenic potential of adipose-derived cells-based therapy. Arterioscler Thromb Vasc Biol 29:1093-1099

40. Calle MC, Fernandez ML (2012) Inflammation and type 2 diabetes. Diabetes Metab 38:183-191

41. Maumus M, Peyrafitte JA, D'Angelo R et al (2011) Native human adipose stromal cells: localization, morphology and phenotype. Int J Obes (Lond) 35:1141-1153

42. Li L, Xie T (2005) Stem cell niche: structure and function. Annu Rev Cell Dev Biol 21:605-631
43. Simon HU, Haj-Yehia A, Levi-Schaffer F (2000) Role of reactive oxygen species (ROS) in apoptosis induction. Apoptosis 5:415418

44. Park SG, Kim JH, Xia Y, Sung JH (2011) Generation of reactive oxygen species in adipose-derived stem cells: friend or foe? Expert Opin Ther Targets 15:1297-1306

45. Noble M, Pröschel C, Mayer-Pröschel M (2011) Oxidativereductionist approaches to stem and progenitor cell function. Cell Stem Cell 8:1-2

46. Garrido AM, Griendling KK (2009) NADPH oxidases and angiotensin II receptor signaling. Mol Cell Endocrinol 302:148-158

47. Waki H, Tontonoz P (2007) Endocrine functions of adipose tissue. Annu Rev Pathol 2:31-56

48. Pradhan AD, Manson JE, Rifai N et al (2001) C-reactive protein, interleukin 6 , and risk of developing type 2 diabetes mellitus. JAMA 286:327-334

49. Fisher G, Hyatt TC, Hunter GR et al (2012) Markers of inflammation and fat distribution following weight loss in African-American and white women. Obesity (Silver Spring) 20:715-720

50. Thörne A, Lönnqvist F, Apelman J, Hellers G, Arner P (2002) A pilot study of long-term effects of a novel obesity treatment: omentectomy in connection with adjustable gastric banding. Int $\mathrm{J}$ Obes Relat Metab Disord 26:193-199 